\title{
Fuzzy Control of Floating Raft Active Vibration Isolation System under Narrowband Interference
}

\author{
Youliang Jiang \\ School of Mechanical and Electronic Engineering, Wuhan University of Technology, Wuhan 430070, China. \\ Hubei Provincial Engineering Technology Research Center for Magnetic Suspension, Wuhan 430070, China.
}

Ruirui Duan

China Ship Development and design center, Wuhan 430064, China.

\author{
Peng Liu \\ School of Mechanical and Electronic Engineering, Wuhan University of Technology, Wuhan 430070, China.
}

(Received 22 April 2020; accepted 1 November 2020)

\begin{abstract}
The harmonic signal generated by power equipment on naval vessels is a typical narrowband interference, which seriously affects the stealth performance of naval vessels. The float raft active vibration isolation system is characterized by strong adaptability and good controllability, which can effectively isolate low-frequency interference. For the active vibration isolation system, the control strategy is one of the core, and the precise mathematical model is difficult to be established. The fuzzy control does not rely on the precise mathematical model of the controlled system, which cannot only provide an intelligent path for the active control of the complex floating raft system, but also effectively suppresses the narrowband interference caused by naval vessels' power equipment. Therefore, depending on the existing float raft active vibration isolation platform, this paper outlines a design for a two-input/single-output optimized fuzzy control system based on vibration acceleration, variation of vibration acceleration and control voltage. In an optimized fuzzy control system, an algorithm of quantum behavior particle swarm optimization algorithm (QPSO) is proposed to optimize quantization factor and scale factor because quantization factor and scale factor are set subjectively with strong subjectivity and blindness, and the stability of the control system is roughly verified. Therefore, simulations and experiments are carried out to test and verify the performance of control system designs outlined in this paper. Through comparative analysis, it shows that the floating raft active vibration isolation system with a fuzzy control system based on the QPSO algorithm has a favorable effect on low-frequency narrowband interference.
\end{abstract}

\section{INTRODUCTION}

To improve the stealth performance of naval vessels, it is significant to reduce naval vessels' mechanical vibration. However, the mechanical vibration of naval vessels is mainly caused by the mechanical narrowband noise generated by mechanical power equipment such as diesel engines and auxiliaries. Vibration isolation technology which can effectively inhibit the energy transfer of mechanical vibration is one of the important research interests in vibration control. ${ }^{1,2}$ Vibration isolation can be divided into passive vibration isolation and active vibration isolation. Passive vibration isolation is limited by fixed parameters, and is disadvantageous to low frequency vibration isolation. Nevertheless, active vibration isolation has the advantages of the dynamic adjustment of parameters, strong adaptability and controllability, and can effectively isolate low-frequency interference. In particular, as one of the significant means to eliminate mechanical narrowband disturbance, the floating raft active vibration isolation can isolate the hull vibration transmitted by power equipment through the pedestal. ${ }^{3-6}$ Research on floating raft active vibration isolation mainly focuses on control strategy. ${ }^{7-11}$ Therefore, relying on the floating raft active vibration isolation system based on an electromagnetic vibration isolator developed by our team, ${ }^{12,13}$ this paper strategizes the designs and studies of the control system utilizing the floating raft active vibration isolation system.

Control strategy is the core of the active vibration isolation system. At present, most control strategies are based on feedback linearization, ${ }^{14}$ which is highly dependent on a system mathematical model. Therefore, it is of practical significance to find a control strategy which does not depend on an accurate mathematical model. Zadeh first proposed the concept of fuzzy control in 1965 and Mamdani first applied fuzzy control to the control system of a steam engine in 1974. ${ }^{15,16}$ Fuzzy control is an intelligent feedback control method that does not rely on the precise mathematical model of the controlled system, and can provide an intelligent path for the active control of complex floating raft active vibration isolation system. ${ }^{17}$ In the fuzzy controller, quantization factor, scale factor, fuzzy control rule and membership function which are the core of fuzzy control and greatly affect the dynamic characteristics the control system, have a certain blindness and subjectivity because these parameters are set subjectively. ${ }^{18,19}$ So, many scholars' research shows that an intelligent algorithm is used to optimize the fuzzy controller.

With the development of the computer, there are now many 
optimization algorithms: genetic algorithm, particle swarm optimization (PSO) algorithm, neural network algorithm, ant colony algorithm etc. ${ }^{7}$ The PSO algorithm is a swarm optimization algorithm put forward by Kennedy and Eberhart in 1995 to simulate birds' predation behavior. ${ }^{20}$ Compared with the intelligent algorithms such as heredity, neural network and ant colony, the PSO algorithm has the advantages of simple structure, fewer parameters and easy realization, thus having been widely used and studied in the fields of function optimization and data mining. Subsequent research and extensive experiments show that the PSO algorithm is prone to premature convergence and falls into a local optimal solution in the iterative process. With the development of particle swarm optimization, a particle swarm optimization algorithm based on quantum theory (QPSO) is proposed. The QPSO algorithm has proven to be a globally optimal intelligent algorithm. ${ }^{21,22}$

Therefore the fuzzy control system based on the QPSO algorithm design will be discussed in this paper, and the performance of the control system will be analyzed and studied in detail. The paper is organized as follows: Section 2 will briefly introduce the existing floating raft active vibration isolation platform and establish the mathematical model by the least squares system identification method for simulation analysis; design and establishment of the control system based on the optimized fuzzy controller are presented in Section 3; Simulation and stability are presented in Section 4, where the quantum behaved particle swarm optimization algorithm (QPSO) is applied to optimize the quantization factor, scale factor and membership function of the fuzzy controller, and the stability of the control system is roughly verified; Section 5 describes the experiment that was conducted to verify whether the proposed control system can effectively restrain the multi-source excitation narrowband disturbance; Lastly, Section 6 summarizes the findings and provides conclusions of this study.

\section{FLOATING RAFT ACTIVE VIBRATION ISOLATION PLATFORM AND MATHEMATICAL MODEL}

The control system designed and studied in this paper is mainly based on the existing floating raft active vibration isolation platform of our team. ${ }^{12,13}$ The existing system is shown in Fig. 1, the left part is a mechanical device and the right part is an electric control device.

The mechanical device includes two adjustable speed exciting motors (1-\#) that are used to simulate vibration signals of power equipment, two motor support plates (2-\#), a floating raft (3), four rubber isolators (3-\#) ( $k$ and $c$ are stiffness and damping), four acceleration sensors (4-\#), four electromagnetic vibration isolators (5-\#), a base (6), etc. The purpose of vibration isolation is to reduce the transmission of vibration. Therefore, the measured values of the acceleration sensors (4\#) can be used not only as feedback error signals, but also as the evaluation index of vibration isolation effect. The smaller the measured values of the sensors are, the better the vibration isolation effect of the system will be, and vice versa. The electric control device mainly includes the conditioning amplifier, low-pass filter, Dspace1103 system, PC, and power amplifier.

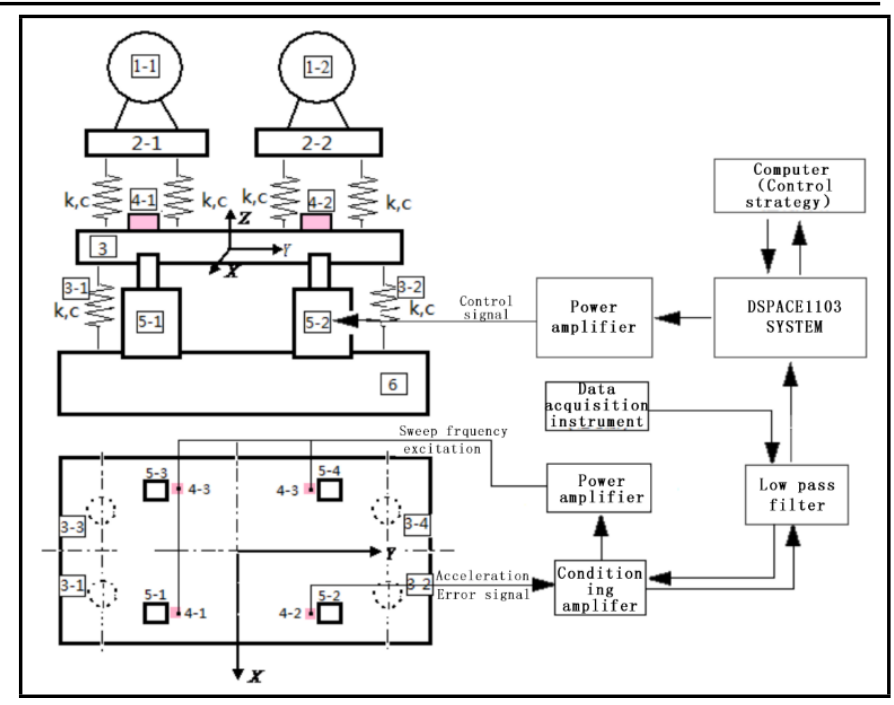

Figure 1. The floating raft active vibration isolation system.

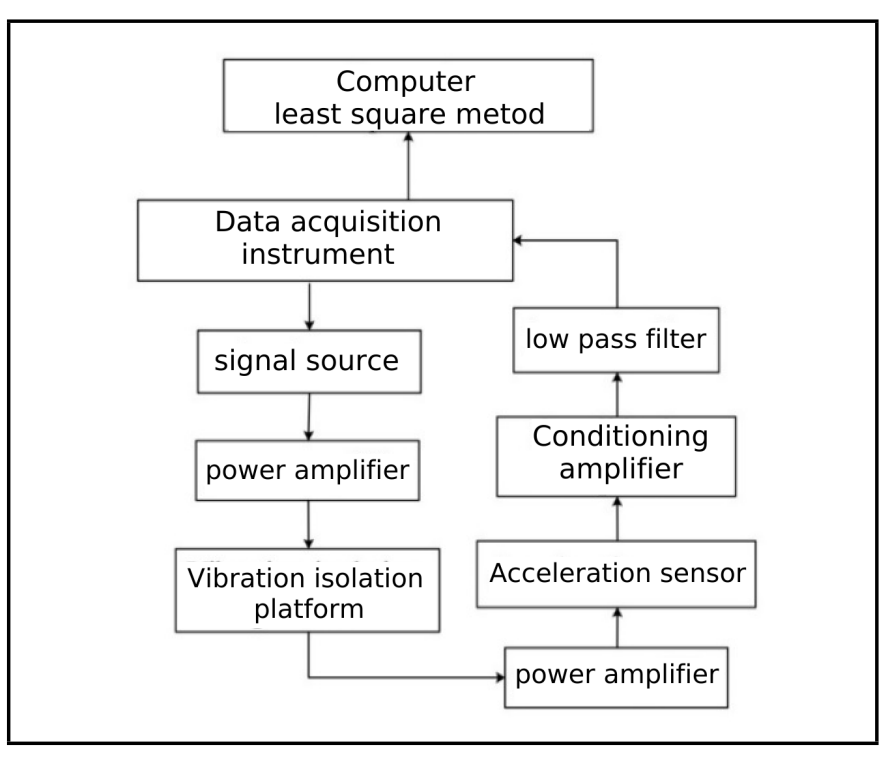

Figure 2. Identification of floating raft active vibration isolation system.

Although the control system designed and studied in this paper does not need a mathematical model in the actual control process, it still depends on the mathematical model in the simulation process. For complex systems, system identification is the main method. Without an in-depth analysis of the system's internal mechanism, system identification is based on the input/output data to identify the identification model of the system. Specifically, the least squares method has the advantages of the simple principle, fast convergence speed, easy realization etc. ${ }^{23}$ Therefore, system identification based on the least squares method is adopted to obtain the mathematical model of the floating raft active vibration isolation platform. Because the power equipment of the ship is not replaced frequently, the off-line identification method is generally used. This paper also adopts the offline method. The acceleration signal (4\#) is taken as the output signal and the signal of the vibration isolator (5\#) as the input signal in the system identification process. Because the narrowband disturbances of the power system mainly range from $15 \mathrm{~Hz}$ to $60 \mathrm{~Hz}$, the frequency range of the identification system is $15 \mathrm{~Hz}-80 \mathrm{~Hz}$. The process is shown in Fig. 2. 
In this paper, different identification models adopt different coherence coefficients and identification orders to eliminate power network interference and achieve a higher fitting degree of identification. Vibration isolators 5-1, 5-3, 5-4 analog disturbance signal channels, and vibration isolator 5-2 are used as the control channel. Whether it is disturbance signal channels or the control channel, the output signal uses the signal of sensor 4-2. On the basis of an input and an output signal, the identification mathematical model from 5-1, 5-2, 5-3, 5-4 to 4-2 can be obtained. The transfer function of the identification mathematical model is shown in Eq. (1)- (4):

$$
S T 12=\frac{\text { num } 12}{\operatorname{den} 12}
$$

where num $12=[0,0.0031,-0.0375,0.1983,-0.6112$, $1.234,-1.7230,1.7033,-1.1935,0.5805,-0.1862,0.0352$, $-0.0030]$ and $\operatorname{den} 12=[1,-10.5212,51.6898,-156.7901$, $327.0369,-494.2043,554.8729,-466.4581,291.4586$, 132.0405, 41.1814, -7.9420, 0.7166];

$$
S T 22=\frac{\text { num } 22}{\operatorname{den} 22}
$$

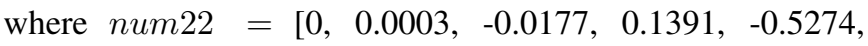
$1.2173,-1.8752,2.0067,-1.5072,0.7831,-0.2689,0.0549$, $-0.0051]$ and $\operatorname{den} 22=[1,-10.2866,49.4444,-146.9165$, $300.7271,-447.0375,495.1921,-412.1290,255.9222$, 115.7002, 36.1625, -7.0183, 0.6398];

$$
S T 32=\frac{\text { num } 32}{\operatorname{den} 32}
$$

where num $32=[0,-0.0008,0.0121,-0.0775,0.2945$, $0.7512,1.3678,-1.8357,1.8417,-1.3818,0.7649,-0.3028$, $0.0808,-0.0129,0.0009]$ and den $32=[1,-12.2697,71.1525$, $-258.3856,656.2571,-1232.9490,1766.8043,-1961.6160$, $1695.6412,-1135.6794,580.2155,-219.3049,57.9852$, 9.6032, 0.7519];

$$
S T 42=\frac{\text { num } 42}{\operatorname{den} 42}
$$

where num $42=[0,0.0044,-0.0577,0.3461,-1.2733$, $3.2099,-5.8626,7.9872,-8.2238,6.4015,-3.7216,1.5708$, $0.4558,0.0815,-0.0068]$ and den $42=[1,-12.1513,69.6614$, $-249.6705,624.9128,-1155.4521,1627.5675,-1774.4318$, $1504.8577,-988.1583,494.6933,-183.1525,47.4263$, 7.6923, 0.5900].

The identification model curve and the experimental model curve are shown in Figs. 3 to 6.

From Figs. 2 to 6 , it is found that the identification model curve and the experimental model curve have a high fitting degree in the frequency domain, especially in the working frequency. The fitting degrees are $95.91 \%, 94.80 \%, 94.61 \%$ and $97.05 \%$ respectively.

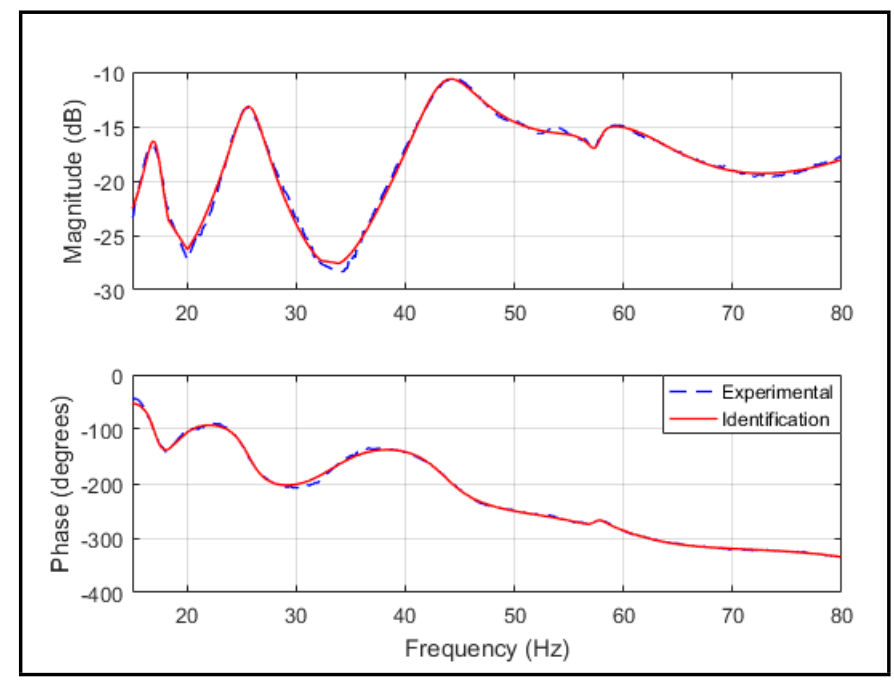

Figure 3. The ST12 experimental model and the identification model.

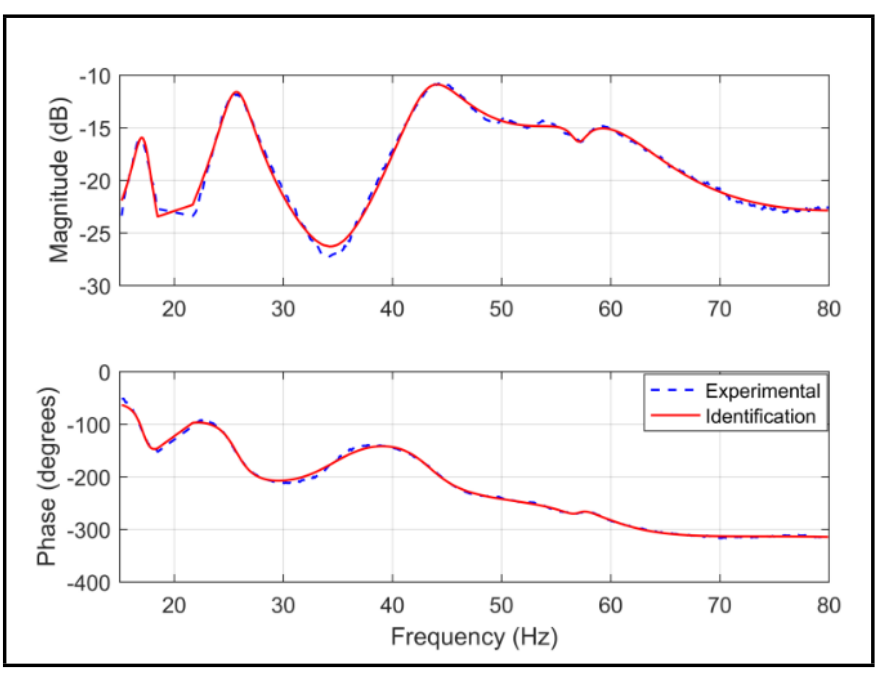

Figure 4. The ST22 experimental model and the identification model.

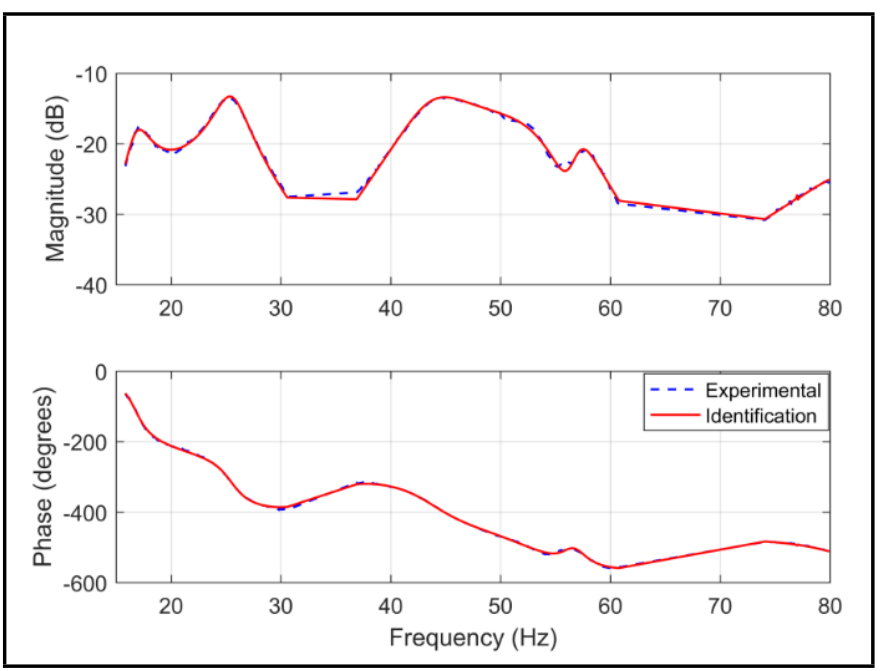

Figure 5. The ST32 experimental model and the identification model. 


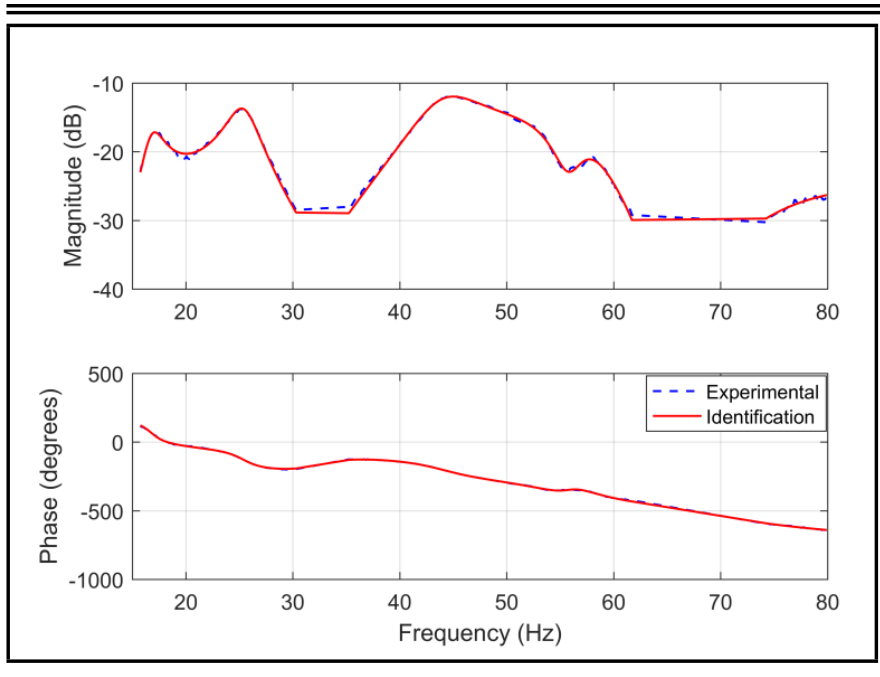

Figure 6. The ST42 experimental model and the identification model.

\section{THE OPTIMIZED FUZZY CONTROL SYSTEM AND THE PARAMETER OPTIMIZATION ALGORITHM BASED ON QPSO}

\subsection{Design of the Optimized Fuzzy Control System}

Because of the influence of nonlinearity, coupling, and hysteresis on the controlled object or the actual system, it is difficult to establish a mathematical model that will accurately describe the dynamic characteristics of the system, thus leading to a huge difference between the actual system and the mathematical model. The fuzzy control, subordinate to intelligent control, is a nonlinear control in essence. ${ }^{24}$ The fuzzy control is not restricted by an accurate mathematical model of the system, and is distinguished and characterized by good adaptability and robustness. It can provide an effective control strategy for a complex floating raft vibration isolation system.

The design of the fuzzy control system mainly includes selecting the input and output of the control system, designing control $p$ establishing the method of fuzzification and defuzzification, determining the domain of variables, selecting the parameters of the fuzzy control system, etc. Quantization factor, scale factor, fuzzy control rule and membership function are at the core of the fuzzy control, and greatly affect the dynamic characteristics of the control system. ${ }^{18,19}$ Generally, the control rules are confirmed by experts' experience with considerable authority while the quantization factor, scale factor and the membership function are set subjectively with strong subjectivity and a blind eye to the selection process. Thus, the quantization factor, the scale factor and the membership function cannot achieve a favorable effect in the actual control, and the optimization of them is one of the most important concerns in the fuzzy control system.

The fuzzy control system based on an intelligent algorithm is a control strategy combined with an artificial intelligence algorithm and the fuzzy control. Through the aid of an artificial intelligent algorithm, the quantization factor, the scale factor and the membership function can be optimized. ${ }^{8,25}$ The fuzzy control system optimized by the intelligent algorithm not only has strong robustness and high reliability, but also has the char-

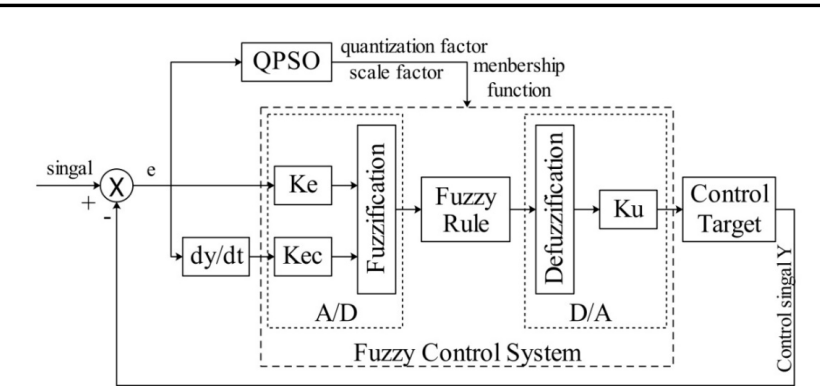

Figure 7. Intelligent optimization of the fuzzy control system structure.

acteristics of an autonomous memory, is self-learning and is self-adaptive. Moreover, it can automatically identify parameters of the controlled process and adapt to the changes of these parameters. Therefore, the intelligent fuzzy control system of a floating raft active vibration isolation system based on an acceleration signal is established in this paper. The design block diagram is shown in Fig. 7.

\subsection{Quantum Behavior Particle Swarm Optimization Algorithm}

The fuzzy control system is based on an artificial intelligence algorithm to automatically adjust the parameters. Compared with other intelligent algorithms, the PSO algorithms have the advantages of a simple structure, fewer parameters and easy realization. The algorithm is prone to premature convergence and falls into a local optimal solution in an iterative process. Therefore, QPSO algorithm which has been proven to be a globally optimal intelligent algorithm, is adopted to optimize the parameters of the fuzzy control in this paper. ${ }^{21}$

The QPSO algorithm is used to describe the state of each particle by using the wave function. The probability density function of a particle at a point in space is first solved by using the Schrodinger equation, and then obtaining the position equation of the particle by the random simulation of the Monte Carlo principle. In the QPSO algorithm, the location updating and evolution equation of particles are as follows:

$$
P=\varphi \times \operatorname{Pbest}(i)+(1-\varphi) \times \operatorname{Gbest}(i)
$$

$$
\begin{array}{r}
\text { mbest }=\frac{1}{M} \sum_{i=1}^{M} \text { Pbest }(i)=\left(\frac{\text { Pbest }(i 1)}{M},\right. \\
\left.\frac{P b e s t(i 1)}{M}, \ldots, \frac{P b e s t(i D)}{M}\right) ;
\end{array}
$$

$$
\operatorname{position}(t+1)=P \pm \alpha \times \mid \text { mbest }-\operatorname{pos}(i) \mid \times \ln \left(\frac{1}{\mu}\right) .
$$

In the above formulas, $M$ is the number of particles, $D$ is particle dimension, Pbest $(i)$ is the optimal position of a particle at the $i$ generation, Gbest $(i)$ is the global optimal position of the population at the $i$ generation, mbest is the average optimal position of the population, position $(t)$ is the relative position coordinate (information) of particles, $\varphi$ and $\mu$ are the 
random numbers of homogeneous distribution between [0,1]. $\alpha$ is a contraction factor. When, $\alpha>0.5$ ' \pm ' takes '-', and vice versa, it takes ' + '.

Due to the fact that quantum theory shows a strong uncertainty in motion, the particles do not have a definite trajectory in quantum space. Because of these characteristics, the QPSO algorithm has a stronger stability and a global convergence than the PSO algorithm. The contraction factor $\alpha$ is the core parameter of the QPSO algorithm, and its value is the key to deciding whether the algorithm converges. Numerous studies conducted by scholars such as Sun have proven that when $\alpha<1.7,{ }^{21}$ the QPSO algorithm converges; when the value varies from 1 to 0.5 , the algorithm has the best convergence effect.

QPSO algorithm flow is as follows:

- Initialize particles.

- Evaluate all particles, put each particle in the best place of Pbest $(i)$, and put the global optimum position in the population in Gbest $(i)$.

- Compare each particle's current value with its fitness value of Pbest $(i)$, and update Pbest $(i)$ by replacing current location and target value if the current target value is better.

- Compare all current Pbest $(i)$ values with the Gbest $(i)$ values and update Gbest $(i)$ if it is better.

- Update particles.

- Judge whether the iteration termination condition is satisfied.

\subsection{Parameters Optimization Based QPSO Algorithm}

In the process of designing the fuzzy control system, the quantization factor $K e$, the scale factor $K e c$ and the membership function are the key parameters determining the performance of the control system. Therefore, the QPSO algorithm is applied to optimize these parameters dynamically so that the floating raft active vibration isolation system can achieve the best vibration isolation effect.

There are many membership functions, such as Gauss type and triangle type. The triangle membership function is adopted in this paper. The scope of its three variables is [-3 3], in which 5 fuzzy partitions (Z1 (Zone 1), Z2 (Zone 2), Z3 (Zone 3), Z4 (Zone 4), NB (Zone 5)) are divided and symmetrically optimized. The membership function of each fuzzy subset is determined by two parameters, as is shown in Fig. 8.

In this paper, a dual input and single output mode was adopted. The vibration acceleration $E$ and its variation $E C$ are taken as the input, and Control voltage $U$ as the output. Therefore, Ea1, Ea2, ECa1, ECa2, Ua1 and Ua2 are the six optimized parameters in the membership function; $K e_{-} E$ and $K e_{-} E C$ are the two optimized parameters in the quantization factor and $K_{e c} U$ is the optimized parameter in the scale factor.

The fitness function of the system is based on the square error integral criterion (ISE): ISE $=\int_{0}^{\infty}|e(t)|^{2} d t$.

The QPSO algorithm and the fuzzy control system are connected by particle coordinate information and fitness

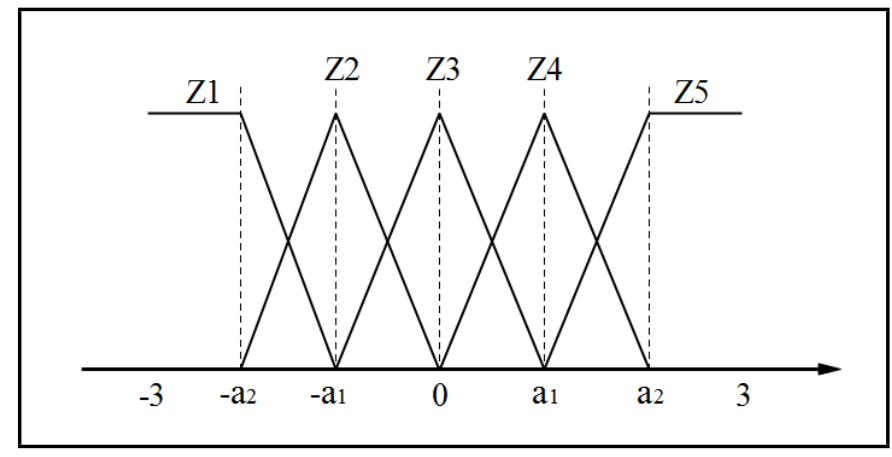

Figure 8. Membership function.

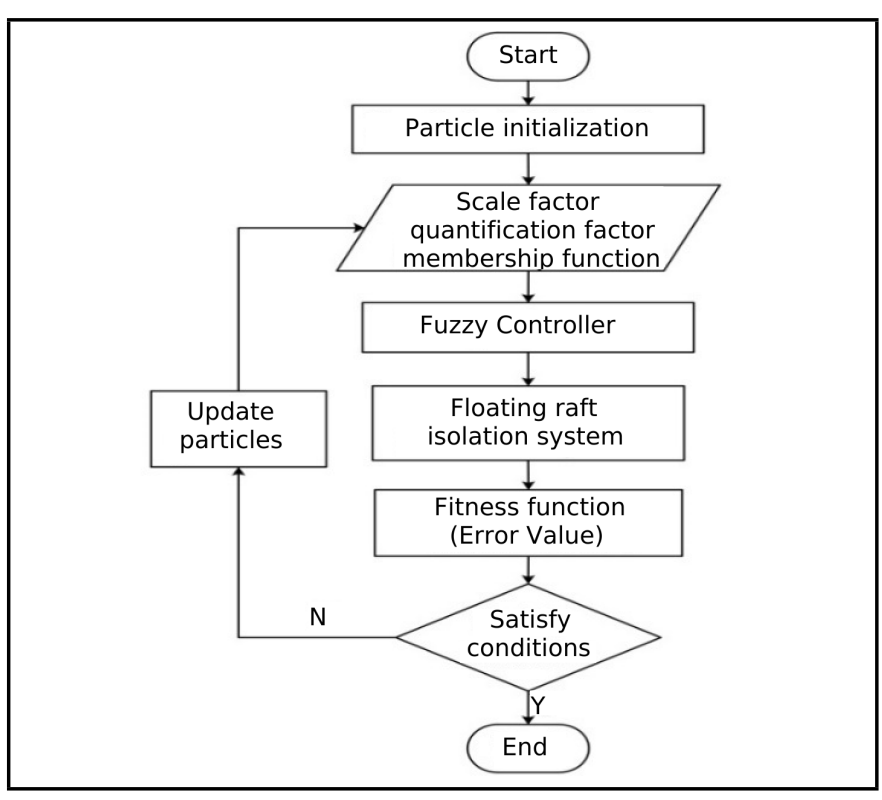

Figure 9. The flowchart of the fuzzy control system optimized by the QPSO algorithm.

value. The coordinate information of the particle stores the parameters to be optimized in the fuzzy control system. The fitness value is the vibration isolation index of vibration isolation system. The coordinate format of the particle is [Ke_E, Ke_eC, Kec_U, Ea1, Ea2, ECa1, Eca2,Ua1,Ua2]. The process of how the QPSO algorithm optimizes the parameters in the fuzzy control system is presented in Fig. 9.

\section{SIMULATION RESEARCH}

\subsection{Simulation Parameters}

This paper mainly studies the vibration isolation effect of the fuzzy control system in a floating raft active vibration isolation platform on narrowband interference signal. Therefore, the simulation analysis is carried out by single source narrowband excitation and multi-source narrowband excitation respectively.

The single sweep frequency $(15 \mathrm{~Hz}-50 \mathrm{~Hz})$, double sweep frequency $(15 \mathrm{~Hz}-30 \mathrm{~Hz}, 25 \mathrm{~Hz}-40 \mathrm{~Hz})$ and multi sweep frequency $(20 \mathrm{~Hz}-35 \mathrm{~Hz}, 30 \mathrm{~Hz}-45 \mathrm{~Hz}, 40 \mathrm{~Hz}-55 \mathrm{~Hz})$ simulate single source, dual source and multi-source narrow-band signals respectively. Those sweep signals simulate the narrowband disturbance generated by system identification models ST12, ST32 and ST42 in the floating raft system. The ST22 is 


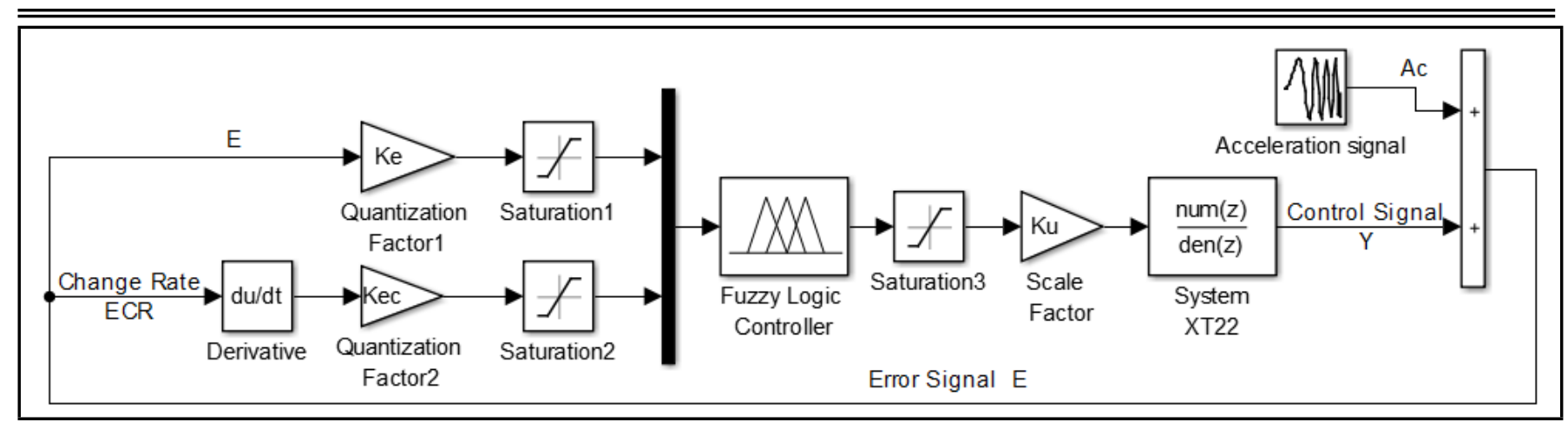

Figure 10. Simulation control diagram of the narrowband disturbance.

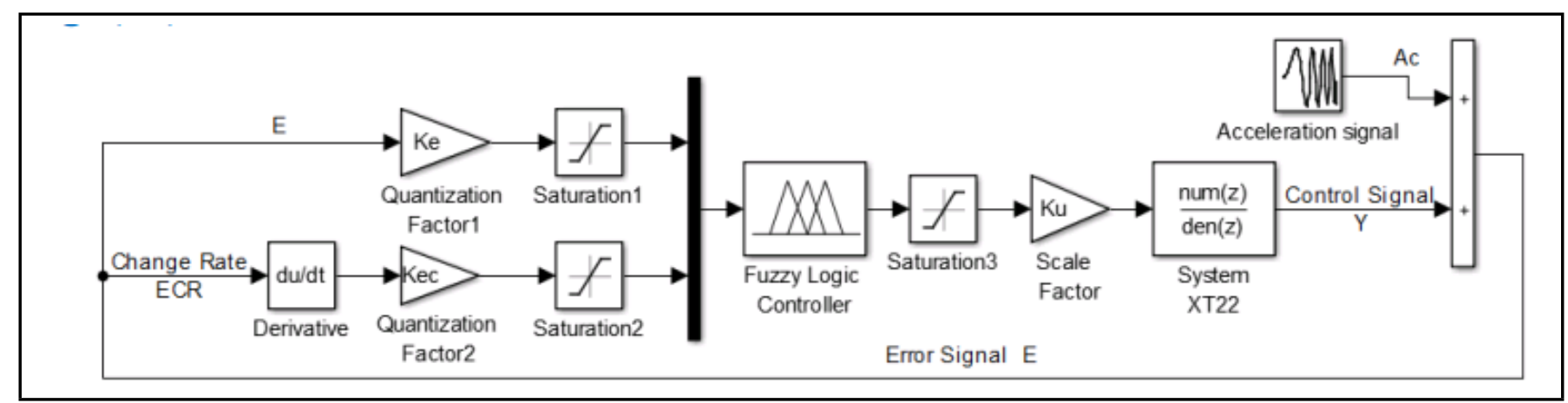

Figure 11. Input and Output of the control system.

Table 1. Control rule table.

\begin{tabular}{|c|c|c|c|c|c|c||}
\hline \hline & & \multicolumn{5}{|c|}{ EC } \\
\hline & $\mathbf{U}$ & $\mathbf{Z 1}$ & $\mathbf{Z 2}$ & $\mathbf{Z 3}$ & $\mathbf{Z 4}$ & $\mathbf{Z 5}$ \\
\hline \multirow{4}{*}{$\mathbf{E}$} & $\mathbf{Z 1}$ & Z5 & Z5 & Z4 & Z4 & Z3 \\
\cline { 2 - 7 } & $\mathbf{Z 2}$ & Z5 & Z4 & Z4 & Z3 & Z3 \\
\cline { 2 - 7 } & $\mathbf{Z 3}$ & Z4 & Z4 & Z3 & Z2 & Z2 \\
\cline { 2 - 7 } & $\mathbf{Z 4}$ & Z3 & Z3 & Z2 & Z2 & Z1 \\
\cline { 2 - 7 } & $\mathbf{Z 5}$ & Z3 & Z2 & Z2 & Z1 & Z1 \\
\hline
\end{tabular}

the mathematical model of a floating raft active vibration isolation platform. The quantization factor, the scale factor and the membership function of fuzzy control system are obtained by the optimization of QPSO algorithm, while the control rules (as seen in Tabl 1) are provided by experts in the field. ${ }^{16}$

Finally, the control system of the floating raft active vibration isolation platform is simulated by the MTALAB/SIMULINK toolbox. The simulation diagram is shown in Fig. 10.

\subsection{The Stability of the Control System}

Stability is one of the important indices of the controller. Since fuzzy control is based on logic judgment rules, there is no transfer function (or state function). Therefore, it is extremely difficult to verify the stability of the fuzzy control system by direct method. The stability of the fuzzy control system is still a significant research field. So, in this paper an accurate method was not verified regarding the stability of the controller, but an approximate method was used to roughly verify its stability.

The approximate mathematical model of the fuzzy control system, obtained by system identification, was used to roughly verify the stability of system. We collected the input/output

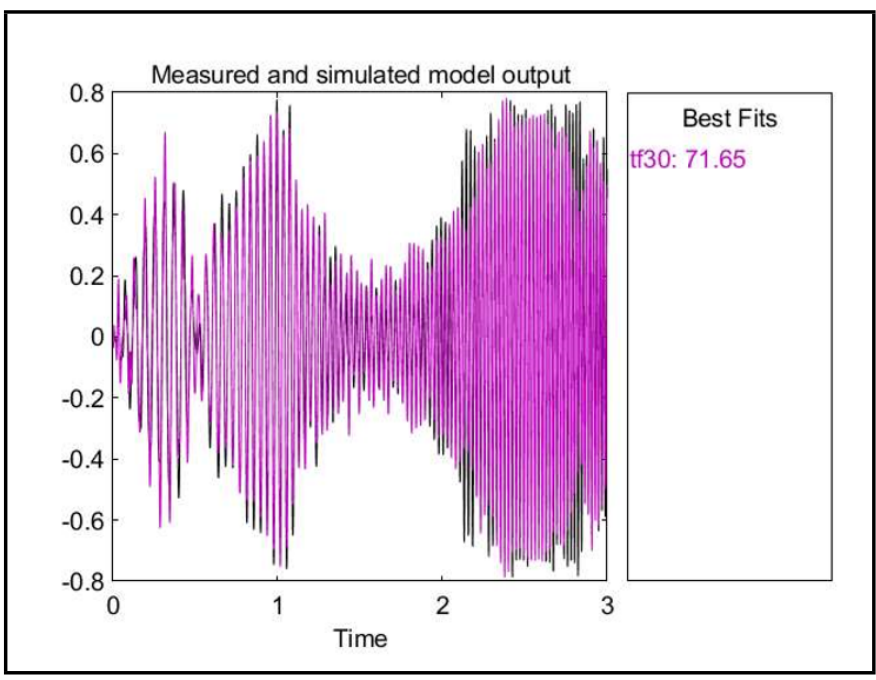

Figure 12. The fitting curve.

data of the control system in the case of system parameters of a single frequency signal. As shown in Fig. 11.

The input/output data were imported into the system identification toolbox (Matlab) for the time domain model identification. By the trial and error method, the fitting degree of the identified model reached $71.65 \%$ (the highest). In this case, the number of poles is 14 and the number of zeros is 10 . The fitting curve is shown in Fig. 12. It cannot accurately replace the control system model, but it approximately expressed the change trend of the model.

The distribution of poles and zeros of the identified mathematical model were obtained to analyze the stability of control system. As shown in Fig. 13, all poles are in the unit circle, so the identified system is stable, and then the stability of the control system was generally verified. 


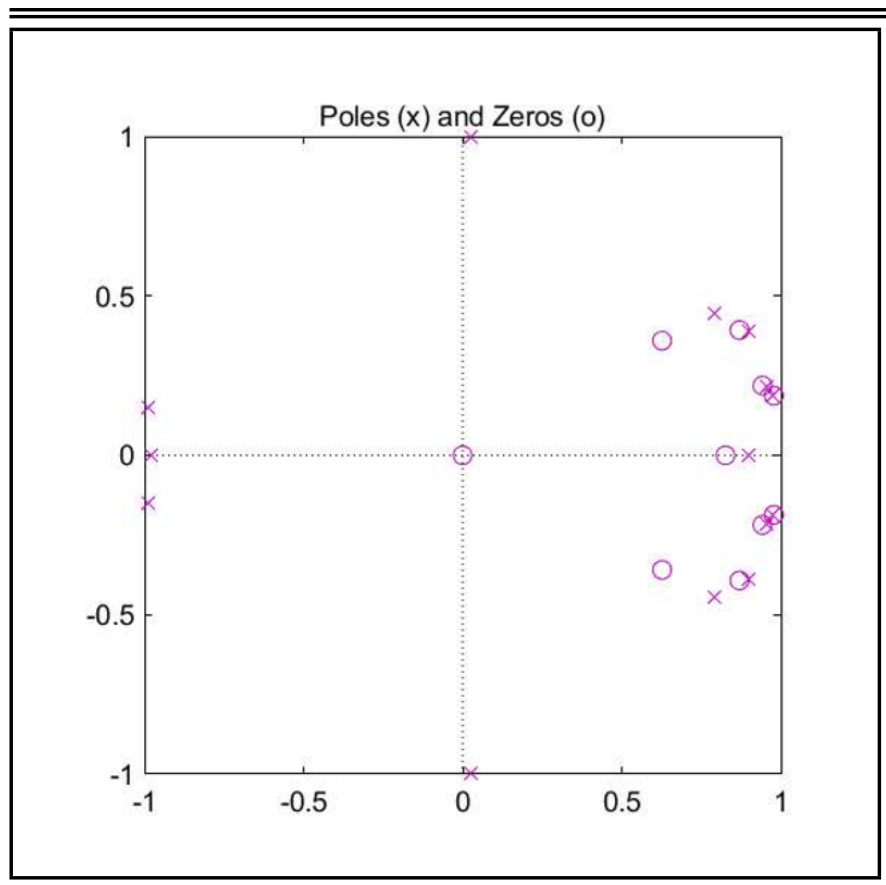

Figure 13. Poles and Zeros.
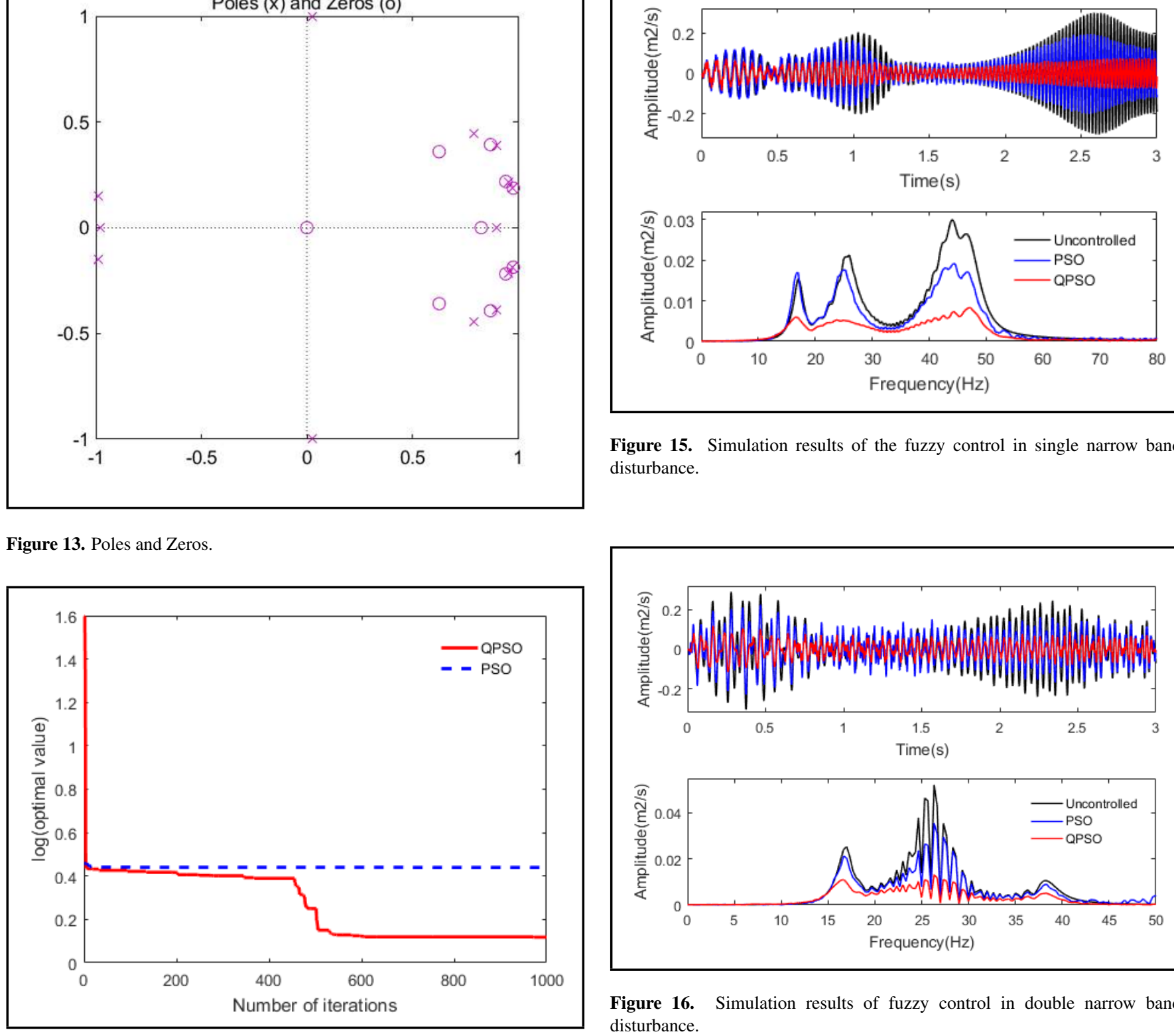

Figure 15. Simulation results of the fuzzy control in single narrow band disturbance.

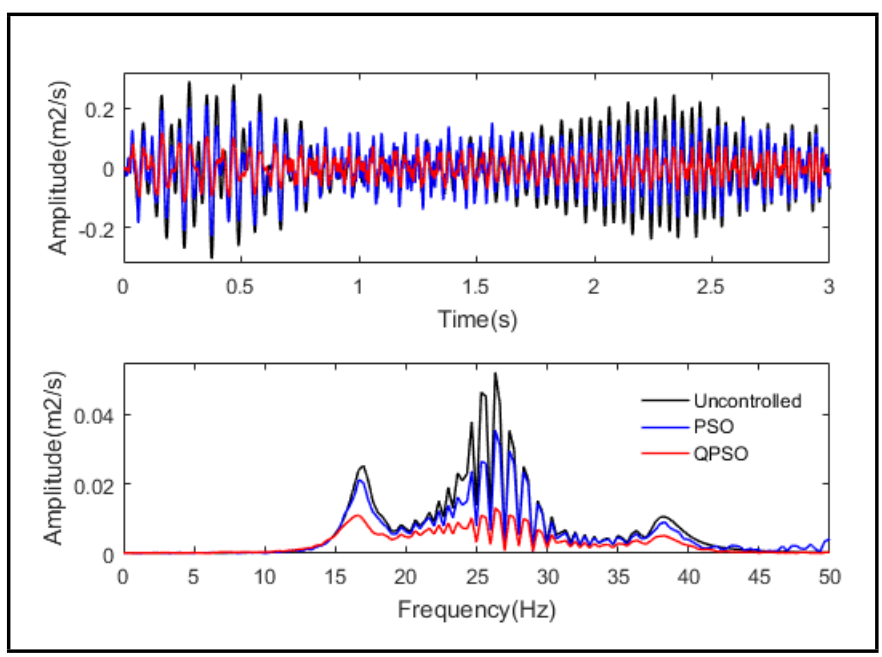

Figure 16. Simulation results of fuzzy control in double narrow band disturbance.

Figure 14. Comparison of QPSO and PSO iteration.

\subsection{Control Simulation Results}

The QPSO algorithm optimizes the quantization factor, scale factor and membership function of the fuzzy control system to get better vibration isolation effect. The initial population of the QPSO algorithm was set as 20, the iteration number was 1000 generations, and the contraction factor $\alpha$ was reduced from 1 to $0.5 .{ }^{20}$ In order to quantitatively analyze and measure vibration isolation effect, the vibration isolation effect in the time and frequency domain was analyzed. On the same condition, the quality of solution between the QPSO algorithm and PSO algorithm was also compared. Figure 14 was an iterative diagram of the fuzzy control system based on QPSO algorithm and PSO algorithm under single source narrowband interference. Figures 15 to 17 are simulation diagrams of the vibration isolation effect of the fuzzy control system based on the QPSO algorithm and the PSO algorithm under three different interferences.

In Fig. 14, both the QPSO algorithm and the PSO algo-
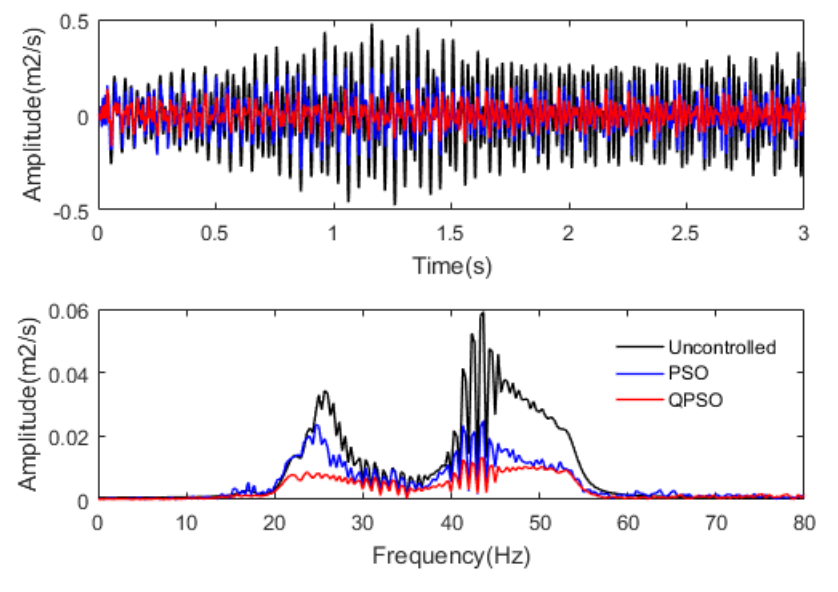

Figure 17. Simulation results of fuzzy control in three narrow band disturbance. 


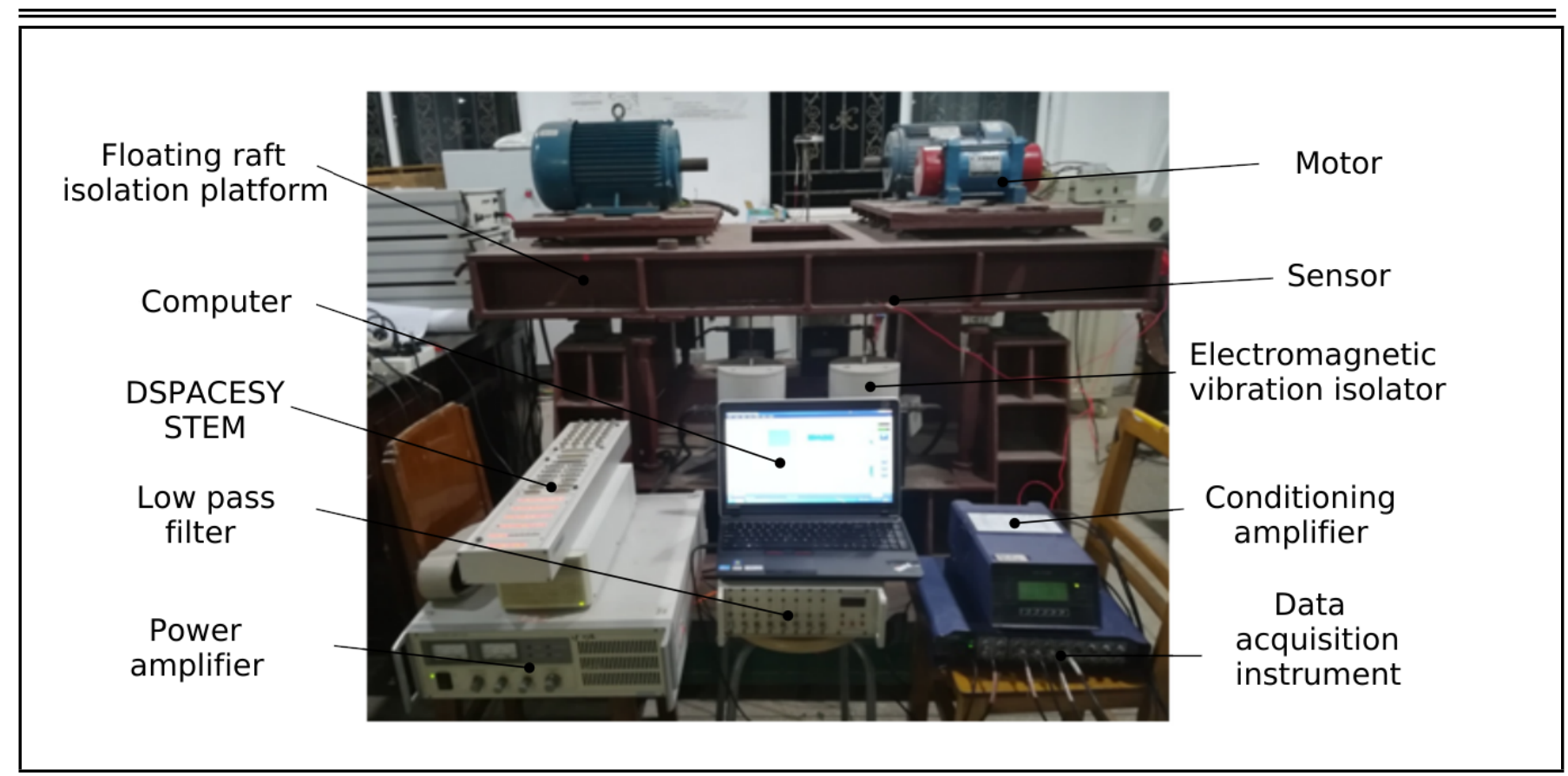

Figure 18. Multi-source excitation floating raft active isolation experiment.

rithm almost reach the local dynamic equilibrium in about the 20th generation. The QPSO algorithm gradually jumps out of the local optimal solution under the random disorder of quantum theory. It reduces dramatically in about the 500th generation. In the 600th generation, the fitness function of the particle reaches the state of dynamic equilibrium, and the optimal particle coordinates are obtained after the operation of the 1000th generation. The QPSO algorithm under each vibration interference is operated by 1000 generations, and the optimized quantization factor, scale factor and membership function are presented below: Single sweep frequency $[17.7711,-0.0215,-0.8554,2.0935,1.4867,2.1449,0.5188$, $2.0704,1.4916]$, double sweep frequency $[6.3413,-0.0088$, $2.1001,2.1176,1.1457,2.0061,1.5,1.8473,1.1523]$, multi sweep frequency $[-8.7068,0.0124,1.8521,1.7937,1.0806$, 2.3888, 1.2017, 1.8590, 1.2377].

Figures 15 to 17 show the acceleration signal of the time and frequency domain in front of and behind the fuzzy control of the vibration isolation system. In Fig. 15, the overall acceleration response amplitude of the fuzzy control system based on PSO algorithm is reduced by $25.68 \%$, and that of the fuzzy control system based on QPSO algorithm is reduced by $61.21 \%$. According to Fig. 16, the overall acceleration response amplitude of the fuzzy control system based on the PSO algorithm is reduced by $21.64 \%$, and that of the fuzzy control system based on the QPSO algorithm is reduced by $48.21 \%$. In Fig. 17, the overall acceleration response amplitude of the fuzzy control system based on the PSO algorithm is reduced by $35.83 \%$, and that of the fuzzy control system based on the QPSO algorithm is reduced by $58.60 \%$.

In summary, the QPSO algorithm is more likely to jump out of the local optimal solution than PSO algorithm. Under three narrowband interference signals, the vibration isolation effect of the fuzzy control system based on the QPSO algorithm has increased more than two times than that based on the PSO algorithm. The QPSO algorithm has better vibration isolation

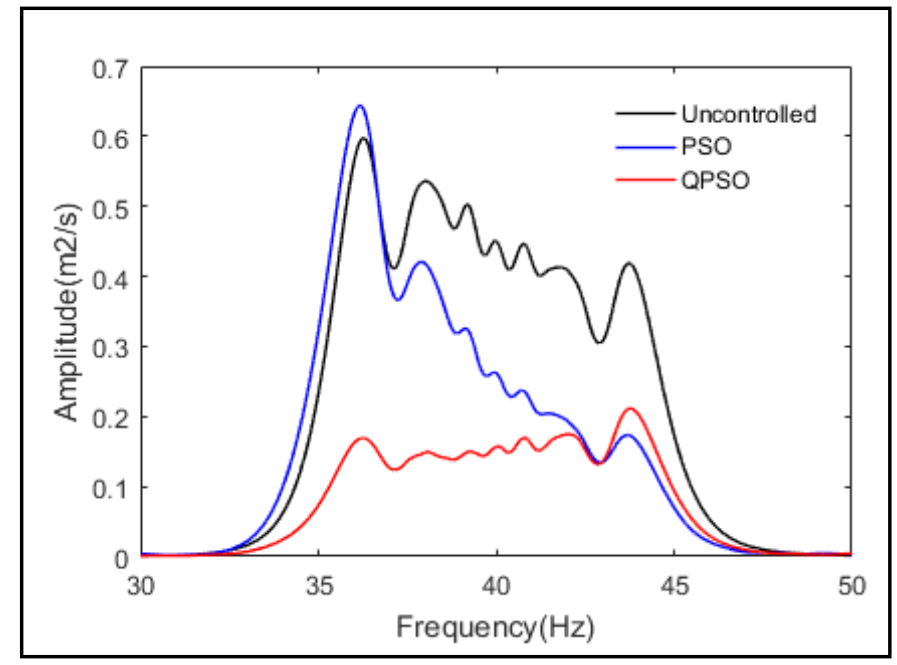

Figure 19. Experimental results of the fuzzy control in single narrowband disturbance.

effect than the PSO algorithm at the multiple main peaks of interference signals, especially at the low-frequency main peaks.

\section{EXPERIMENTAL ANALYSIS}

In order to verify whether the proposed control system can effectively restrain the multi-source excitation narrowband disturbance, a multi-source excitation floating raft active vibration isolation platform was constructed, and control experiments on the narrow band disturbance were carried out. The experiment platform and control experiment are shown in Fig. 18.

In the experiment, the parameters setting of the control system is coincident with that of the simulation, and the frequency and type of interference signals are the same as those of the simulation. The performance of the vibration isolation was analyzed by the frequency domain method. Figs. 19 to 21 shows the experimental results of the vibration isolation effect of the fuzzy control system based on the QPSO algorithm and the PSO algorithm under three different interferences. 


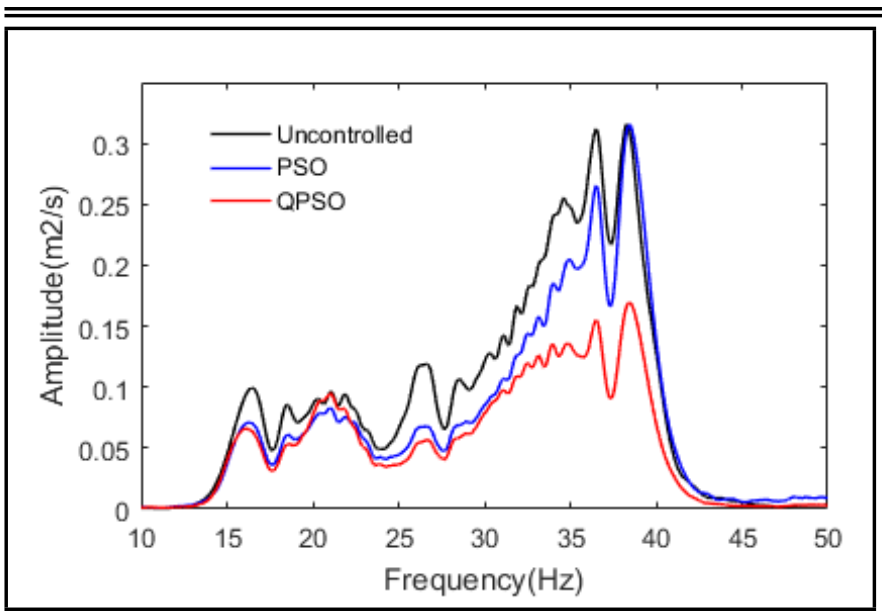

Figure 20. Experimental results of the fuzzy control in double narrowband disturbance.

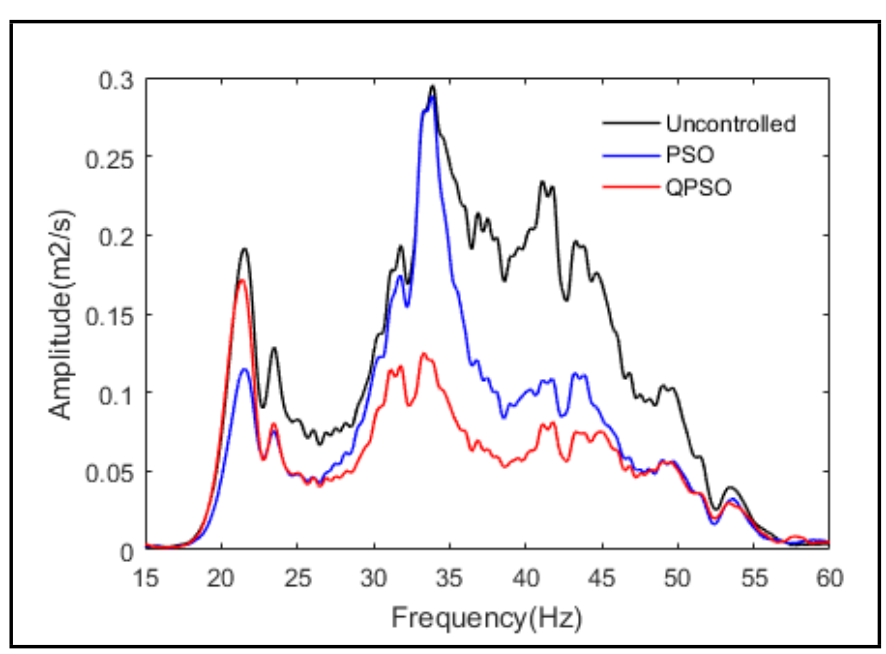

Figure 21. Experimental results of the fuzzy control in three narrowband disturbances.

Figures 19 to 21 show the frequency domain diagram of the acceleration signal in front of and behind the fuzzy control of the vibration isolation system. In the experimental analysis of Fig. 19, the overall acceleration amplitude of the fuzzy control system based on the PSO algorithm is reduced by $32.54 \%$, and that of the fuzzy control system based on the QPSO algorithm is reduced by $63.54 \%$. As seen in Fig. 20, the overall acceleration amplitude of the fuzzy control system based on the PSO algorithm is reduced by $21.08 \%$, and that of fuzzy control system based on the QPSO algorithm is reduced by $35.08 \%$. As is shown in Fig. 21, the overall acceleration amplitude of the fuzzy control system based on the PSO algorithm is reduced by $35.08 \%$, and that of fuzzy control system based on the QPSO algorithm is reduced by $46.10 \%$. In summary, although in the lower part of the vibration types and frequency band, the fuzzy control system optimized by the QPSO algorithm has no better vibration isolation effect than the PSO algorithm as its overall vibration isolation effect is increased by nearly 1.5 times. Therefore, the validity and performance of the proposed control strategy can be verified.

\section{CONCLUSION}

Due to the complexity of the floating raft active isolation system, and the difficulty to establish an accurate mathematical model, a fuzzy control strategy optimized by the QPSO algorithm to control the floating raft active vibration isolation system was proposed in this paper. First, a control system based on an intelligent fuzzy control system was established. Then, the QPSO algorithm was proposed to optimize the quantization factor, scale factor and membership functions of the fuzzy control system. The stability of the control system is roughly proven by the approximate method. Finally, simulations and experiments were conducted under narrowband interference signals of different types and bandwidth. The vibration isolation effect of the experiment was not as good as that of the simulation because simulation was conducted in an ideal state and the experiment was influenced by many external interferences. However, its effect on vibration isolation was still remarkable. The results show that the fuzzy control system based on the QPSO algorithm reduced the amplitude of vibration by $50 \%$ in front and behind, and the vibration isolation effect of the fuzzy control system based on QPSO algorithm in a floating raft active vibration isolation system was better than that based on the PSO algorithm. Therefore, the fuzzy control system based on the QPSO algorithm can effectively solve problems caused by difficult modeling and controlling of the floating raft active vibration isolation system, and improve the performance and application of the fuzzy control system.

\section{DATA AVAILABILITY}

The data used to support the findings of this study have been included within the article.

\section{ACKNOWLEDGEMENTS}

This work was supported by the National Natural Science Foundation of China (No. 51879209), the National Key Program of China (No. 2018YFB2000103) and the Fundamental Research Fund for the Central Universities (China, No. 2020YB-026, No. 185204008, No. 195204016).

\section{COMPETING INTERESTS}

The authors declare that there are no conflicts of interest regarding the publication of this paper.

\section{REFERENCES}

1 Jiang, G., Shen, R., Hua, H., and Wu, G. Advances in study on shock isolation of naval equipment, Journal of Ship Mechanics, 10(1), 135-144, (2006). https://dx.doi.org/10.3969/j.issn.1007-7294.2006.01.018.

2 Liu, Z. Development Trend of the Naval Vessels Stealth Techology, Ship Electronic Engineering, 34(3), 25-29, (2014).

3 Song, C. Study on Theory and Control Technology of Magnetic Suspension Active Vibration Isolation for Flexible Floating, Wuhan University of Technology, Wuhan, Hubei, (2011). https://dx.doi.org/10.7666/d.y1948884. 
4 Wu, C., and Du, K. Design Method of Floating Raft Isolation System, Naval Engineering Research, 37-45, (1996).

5 Zhang, H., and Fu, Z. The Effects of Parameters of Floating Raft Isolation System on Its Isolation Characteristics, Journal of Vibration and Shock, 19(2), 5-8, (2000). https://dx.doi.org/10.3969/j.issn.1000-3835.2000.02.002.

6 Zhou, L., Yang, T., and Zhang, P. Tests for an active floating raft vibration isolation system based on a flexible hull structure, Journal of Vibration and Shock, 32(17), 145-149, (2013). https://dx.doi.org/10.3969/j.issn.10003835.2013.17.028.

7 Gosiewski, Z., and Mystkowski, A. Robust control of active magnetic suspension: Analytical and experimental results, Mechanical Systems \& Signal Processing, 22(6), 1297-1303, (2008). https://dx.doi.org/10.1016/j.ymssp.2007.08.005.

8 Van der Sande, T. P. J., Gysen, B. L. J., Besselink, I. J. M., Paulides, J. J. H., Lomonova, E. A., and Nijmeijer, H. Robust control of an electromagnetic active suspension system: Simulations and measurements, Mechatronics, 23(2), 204-212, (2013). https://dx.doi.org/10.1016/j.mechatronics.2012.07.002.

9 Muradova, A., and Stavroulakis, G. Fuzzy Vibration Control of a Smart Plate, International Journal for Computational Methods in Engineering Science \& Mechanics, 14(3), 212-220, (2013). https://dx.doi.org/10.1080/15502287.2012.711427.

10 Yang, S. M. Electromagnetic Actuator Implementation and Control for Resonance Vibration Reduction in Miniature Magnetically Levitated Rotating Machines, IEEE Transactions on Industrial Electronics, 58(2), 611-617, (2011). https://dx.doi.org/10.1109/TIE.2010.2046000.

11 Pham, M., and Ahn, H. A horizontal vibration isolator with electromagnetic planar actuator using multi hall-effect sensor network, International Conference on Ict Convergence, Jeju Island, (2013).

12 Yang, B., Hu, Y., Vicario, F., Zhang, J., and Song, C. Improvements of Magnetic Suspension Active Vibration Isolation for Floating Raft System, International Journal of Applied Electromagnetics and Mechanics, 53, 193-209, (2017). https://dx.doi.org/10.3233/JAE-150167.

13 Song, C., Yu, C., Xiao, Y., and Zhang, J. Fuzzy logic control based on genetic algorithm for a multi-source excitations floating raft active vibration isolation system, Advances in Mechanical Engineering, 9(6), 168781401770584, (2017). https://dx.doi.org/10.1177/1687814017705843.

14 Lindlau, J. D., and Knospe, C. R. Feedback linearization of an active magnetic bearing with voltage control, IEEE Transactions on Control Systems Technology, 10(1), 21-31, (2002). https://dx.doi.org/10.1109/87.974335.
15 Jenkins, D. F., and Passino, K. M. An introduction to nonlinear analysis of fuzzy control systems, Journal of Intelligent and Fuzzy Systems, 7(1), 75-103, (1999). https://dx.doi.org/10.1080/095281399146643.

16 Collins, E. G. Fuzzy control systems design and analysis: A linear matrix inequality approach, Automatica, 39(11), 2011-2013, (2003). https://dx.doi.org/10.1016/s00051098(03)00188-2.

17 Coelho, L. S. Novel Gaussian quantum-behaved particle swarm optimiser applied to electromagnetic design. IET Science Measurement Technology, 1(5), 290-294, (2007). https://dx.doi.org/10.1049/iet-smt:20060124.

18 Esmin, A. A. A., and Aoki, A. R. Lambert-Torres, G. Particle swarm optimization for fuzzy membership functions optimization, IEEE International Conference on Systems, Washington, DC, (2003).

19 Kaplan, A., Ozer, S., and Sagiroglu, S. Membership function optimization of a fuzzy controller using modified tabu search algorithm, Fuzzy Information Processing Society - NAFIPS, Conference of the North American, Pensacola Beach, Florida, (1998).

20 Kennedy, J., and Eberhart, R. Particle swarm optimization, IEEE International Conference on Neural Networks, the University of Western Australia, Perth, (1995). https://dx.doi.org/10.1007/978-0-387-30164-8_630.

21 Jun, S., Bin, F., and Wenbo, X. Particle swarm optimization with particles having quantum behavior, IEEE 2004 Congress on Evolutionary Computation, Portland, OR, (2004).

22 Fang, W., Sun, J., Ding, Y., Wu, X., and Xu, W. A Review of Quantum, Iete Technical Review, 27(4), 336-348, (2010). https://dx.doi.org/10.4103/0256-4602.64601.

23 Kashyap, R. System identification, IEEE Transactions on Automatic Control, 18(1), 85-86, (1973). https://dx.doi.org/10.1109/TAC.1973.1100195.

${ }^{24}$ Fu, J., Li, P., Wang, Y., Liao, G., and Yu, M. Modelfree fuzzy control of a magnetorheological elastomer vibration isolation system: analysis and experimental evaluation, Smart Materials and Structures, 25(3), 035030, (2016). https://dx.doi.org/10.1088/0964-1726/25/3/035030.

25 Qiu, Z. C., Ling, D. F., Zhang, X. M., and Han, J. D. Vibration control of two-connected piezoelectric flexible plate using nonlinear algorithm and T-S fuzzy controller, Journal of Intelligent Material Systems \& Structures, 26(2), 219-243 (2014). https://dx.doi.org/10.1177/1045389X14523857. 